\title{
The future of precision medicine in opioid use disorder: inclusion of patient-important outcomes in clinical trials
}

\author{
Nitika Sanger, ${ }^{1 *}$ Balpreet Panesar, ${ }^{2 *}$ Tea Rosic, ${ }^{3}$ Brittany Dennis, ${ }^{4}$ Alessia D’Elia, ${ }^{2}$ Alannah \\ Hillmer, ${ }^{2}$ Caroul Chawar, ${ }^{2}$ Leen Naji, ${ }^{5}$ Jacqueline Hudson, ${ }^{3}$ M. Constantine Samaan, ${ }^{6,7,8}$ \\ Russell J. de Souza, ${ }^{7,9}$ David C. Marsh, ${ }^{10}$ Lehana Thabane, ${ }^{7}$ Zainab Samaan ${ }^{3,7,9,11}$ (iD \\ ${ }^{1}$ Medical Science Graduate Program, McMaster University, Hamilton, ON, Canada. ${ }^{2}$ Neuroscience Graduate Program, McMaster University, \\ Hamilton, ON, Canada. ${ }^{3}$ Department of Psychiatry and Behavioural Neurosciences, McMaster University, Hamilton, ON, Canada. ${ }^{4}$ Department \\ of Medicine, McMaster University, Hamilton, ON, Canada. ${ }^{5}$ Department of Family Medicine, Halton Healthcare, Milton, ON, Canada. \\ ${ }^{6}$ Department of Pediatrics, McMaster University, Hamilton, ON, Canada. ${ }^{7}$ Department of Health Research Methods, Evidence, and Impact, \\ McMaster University, Hamilton, ON, Canada. ${ }^{8}$ Michael G. DeGroote School of Medicine, McMaster University, Hamilton, ON, Canada. \\ ${ }^{9}$ Population Genomics Program, Chanchlani Research Centre, McMaster University, Hamilton, ON, Canada. ${ }^{10}$ Northern Ontario School of \\ Medicine, Laurentian University, Sudbury, ON, Canada. ${ }^{11}$ Clinician Investigator Program, Faculty of Health Sciences, McMaster University, \\ Hamilton, ON, Canada. * These authors contributed equally to this article.
}

\begin{abstract}
Objective: Opioid use has reached an epidemic proportion in Canada and the United States that is mostly attributed to excess availability of prescribed opioids for pain. This excess in opioid use led to an increase in the prevalence of opioid use disorder (OUD) requiring treatment. The most common treatment recommendations include medication-assisted treatment (MAT) combined with psychosocial interventions. Clinical trials investigating the effectiveness of MAT, however, have a limited focus on effectiveness measures that overlook patient-important outcomes. Despite MAT, patients with OUD continue to suffer negative consequences of opioid use. Patient goals and personalized medicine are overlooked in clinical trials and guidelines, thus missing an opportunity to improve prognosis of OUD by considering precision medicine in addiction trials.

Methods: In this mixed-methods study, patients with OUD receiving MAT ( $n=2,031$, mean age 39.1 years [SD 10.7], 44\% female) were interviewed to identify patient goals for MAT.

Results: The most frequently reported patient-important outcomes were to stop treatment (39\%) and to avoid all drugs (25\%).

Conclusion: These results are inconsistent with treatment recommendations and trial outcome measures. We discuss theses inconsistencies and make recommendations to incorporate these outcomes to achieve patient-centered and personalized treatment strategies.
\end{abstract}

Keywords: Opioid; outcomes; clinical trials; patient important

\section{Introduction}

Substance use disorder is a chronic and complex behavior with multifaceted health and social consequences. Prescription opioid misuse has become a public health crisis in the United States and Canada, with its reach spreading to other societies at a global level. ${ }^{1-3}$ The root and progression of the opioid crisis in North America have been covered in all types of media as the crisis has touched the lives of many, and its detrimental effects are seen daily in the form of increased mortality and healthcare utilization. In a recent outlook on the rationale for opioid overprescription patterns that began in the 1980s and have continued since, managing pain was found to be the catalyst for the wide distribution of opioids, based on weak evidence contained in a letter to the editor published in the New England Journal of Medicine. ${ }^{4,5}$ Nonetheless, the rate of opioid prescribing and use continues to rise,

Correspondence: Zainab Samaan, Clinician Investigator Program, Faculty of Health Sciences, McMaster University, 100 West 5th St., Hamilton, ON, L8N 3K7, Canada.

E-mail: samaanz@mcmaster.ca

Submitted Sep 30 2019, accepted Apr 17 2020, Epub Jun 122020. leading to an increased incidence of opioid use disorder (OUD). A report compiled by the Substance Abuse and Mental Health Services Administration (SAMHSA) found that over 2.1 million people in the United States are suffering from an OUD related to prescription opioids. ${ }^{6}$

OUD is a chronic, relapsing disorder that effects all aspects of an individual's life - physical, social, and psychological. ${ }^{7}$ A central feature of OUD are the withdrawal symptoms that are experienced when opioids are abruptly stopped, or their dose reduced. Examples of these symptoms are sweating, agitation, shakes, and muscle pains. ${ }^{7}$ Research has also suggested that the severity of withdrawal symptoms experienced may be associated with why patients who are receiving treatment for OUD relapse. ${ }^{8}$

There are various treatment options available for OUD, which are usually a combination of psychological and pharmacological interventions. The pharmacological intervention includes medication-assisted treatment (MAT), which

How to cite this article: Sanger N, Panesar B, Rosic T, Dennis B, D'Elia A, Hillmer A, et al. The future of precision medicine in opioid use disorder: inclusion of patient-important outcomes in clinical trials. Braz J Psychiatry. 2021;43:138-146. http://dx.doi.org/10.1590/15164446-2019-0734 
can include opioid agonists, partial agonists, and antagonists. ${ }^{9,10}$ One of the most common types of MAT is methadone maintenance treatment (MMT). Methadone is a synthetic opioid that can have long-lasting effects for up to 24 hours $^{9}$ and helps to alleviate withdrawal symptoms, usually without the euphoric effects associated with opioids. ${ }^{9}$ While studies have shown that MMT is effective, there is still great variability in treatment response, ${ }^{11,12}$ and outcome measures to assess the effectiveness of methadone are inconsistent. ${ }^{13}$

As OUD can affect people in multiple ways, including physical and mental health, social impact, economic burden, quality of life, and life expectancy, it is therefore difficult to identify which of these aspects clinical trialists, healthcare services, and providers should focus on when developing treatment programs. There are many challenges to consider when deciding on an outcome measure for a chronic disorder with multifaceted impact such as OUD. There is also a need to consider what patients want as a successful and desirable treatment outcome for them to ensure better prognosis and implement a personalized medicine approach. More specifically, the challenges that need to be addressed include how a personalized medicine approach impacts MAT clinical trials and guideline recommendations. Important questions to consider in tackling this challenge include: what is an outcome of treatment success, who selects the desired outcome? How should treatment programs be evaluated? What is the best use of limited healthcare and social-services resources in managing OUD? How do personal characteristics affect treatment outcomes? And finally, how might addressing these challenges support incorporation of precision medicine into addiction clinical trials?

Guidelines for the management of OUD make recommendations for treatment based on findings from clinical trials, expert opinions, and literature reviews. Guidelines strongly recommend the use of MAT to reduce opioid use and/or retain patients in treatment. ${ }^{14}$ These strong recommendations and the selected outcomes do not consider patient-important goals or the different sociodemographic profiles of patients. Thus, these guidelines are based on the notion that the same treatment is recommended for every patient. Although these recommendations and treatment outcomes are important and reduce harm for many patients with OUD, there remains an important aspect of patient-relevant treatment goals, such as a focus on personalized treatment, that is not being considered in current evidence-based practice.

The overwhelming variation in the selection of MAT outcomes in trials, as well as the lack of inclusion of patient-important outcomes in current guidelines, demand further research to establish a set of treatment outcomes that considers patients' goals and preferences. This will allow future trials to measure the effectiveness of MAT and tailor treatment recommendations based on personalized profiles to improve OUD prognosis and move toward precision medicine in clinical trials of addiction treatment.

Within this context, the objectives of this study were: to identify treatment goals of patients with OUD receiving MAT; and to investigate if there are differences in patient- reported treatment goals by age, sex, gender, ethnicity, employment, treatment duration, and type of treatment received.

Our ultimate purpose is to provide suggestions for the inclusion of patients' goals (patient-important outcomes) in clinical trials as a way of promoting the use of precision medicine in managing OUD.

\section{Methods}

This is a mixed-methods study, using qualitative and quantitative data collection and analyses.

\section{Eligibility criteria}

Participants were eligible for this study if they were 16 years of age or older, if they fulfilled the DSM- 5 criteria for OUD, were receiving MAT for OUD at the time of recruitment, and provided written informed consent.

\section{Data collection}

Data were part of a large research program designed to investigate factors associated with OUD. The current study is a primary study that was planned a priori within a large program of OUD-related research. Participants were recruited from community-based addiction treatment centers in Canada and interviewed face-to-face at these centers by research personnel. Data collection for this study occurred between May 2018 and August 2019. The data collected included sociodemographic details, current and past substance use, and psychological and physical health symptoms using structured questionnaires. Demographic information included age, gender (social construct), sex (biological construct), ethnicity, marital status, employment, education, and MAT. Urine drug screen results for the past 3 months were collected at the time of study enrollment. Study participants were also asked an open-ended question: "What are your goals of treatment?" Answers related to this question were written by research personnel at the time of the interview in a free text format, with no restrictions on text length or content.

\section{Quantitative statistical methods}

The participants' demographic information was summarized using descriptive summary measures, expressed as mean (standard deviation) or median (minimum-maximum) for continuous variables and number (percent) for categorical variables.

Patient-important outcomes were defined on the basis of participants' goals and were compared by six variables: age, sex, gender, ethnicity, employment, and type of current MAT. Age was trichotomized into age groups defined by Statistics Canada ${ }^{15}$ : "youth" (16-24 years), "adults" (25-64 years), and "senior" (65+). Sex was coded as male, female, and intersex. Gender was coded as cisgender male, cisgender female, and other (transgender male, transgender female, two-spirit, non-binary, genderfluid, genderqueer, and agender, as reported by participants in response to the question "what gender do you most identify with?"). 
Ethnicity was self-reported by participants and was coded as European, East Asian (Chinese, Japanese, Malaysian, Korea, Papua New Guinea, Thailand, Philippines, Indonesia, Vietnam, Cambodia, Laos, Myanmar/Burma, Bhutan, Singapore), Persian and Arab, African, South Asian (Indian, Sri Lanka, Pakistan, Nepal, Bangladesh), Indigenous (Native North American, Native South or Latin American, Australian Aborigine), and other/ mixed. Employment was coded as currently working or not working. Type of treatment was defined as methadone, buprenorphine/naloxone (Suboxone), or other.

\section{Qualitative data analysis methods}

Nvivo 12 qualitative data analysis software (QSR International) was used to perform a deep-level analysis of the participants' treatment goal response data. ${ }^{16}$ The data management and analyses plans are described in steps 1-3.

\section{Step 1: cleaning and importing the data}

For qualitative analysis, data were first cleaned in Microsoft Excel to minimize typographical errors present in the free-text responses to the question asking participants about their treatment goals. The data were imported into Nvivo, with the text pertaining to participant goals imported as an open-ended question while attribute-assigning data, such as age and sex, were imported as closedended questions. The latter are not codable in Nvivo, and were not analyzed using this software.

\section{Step 2: word frequency query and text search queries}

The free-text data were run through a word frequency query to logically arrange the information and determine the most common four-letter words. The words that occurred most frequently were considered to be representative of the participants' perspectives, as it is assumed that important and significant words are used more often. ${ }^{17}$ The word count query helped identify initial patterns in the data, and there is evidence that this function improves analytic accuracy when compared to manual qualitative word frequency analyses. ${ }^{17}$ In order to avoid decontextualization of the free-text answers, the minimum number of letters permissible in the word frequency query was four. Any word with a frequency weighting of greater than $0.5 \%$ was coded as a node. A node is a collection of references found in the free-text data that corresponds to a particular theme or word. ${ }^{18}$ Words with a word frequency percentage above $0.5 \%$ that were related to a similar theme were grouped in the same node. Words with word frequency percentages above $0.20 \%$ were scanned and included in existing nodes with which they shared similarities.

The text search query allows words and their stemmed variants to be identified as references found in the freetext data responses. Text search queries were conducted for words identified in the word frequency queries to identify related-stemmed words. Results from the text search query were then coded into the appropriate nodes. Patterns and coding strategies emerged as a result of grouping similar words into nodes; these nodes were then labelled as themes.

\section{Step 3: matrix coding queries}

Matrix coding queries help compare participant responses across and between different demographic categories. ${ }^{18}$ Before comparing demographic categories, this query was run between coded references (text that had already been coded at a node) and participant responses, to identify any responses that had not been coded at a node. If a participant had a free-text response but was missing a corresponding coded reference at any of the different nodes, the free-text response was reviewed, and a reference was added to the appropriate node. This process brought forth new words and themes that were eventually combined with existing nodes. Any new words that were identified were also put through a text search query to ensure all the stemmed words were identified and coded into a node. The process of conducting a matrix query to identify any missing references and new/stemmed words was completed iteratively until all participant responses had a coded reference(s).

Another matrix coding query was run between different demographic categories and the nodes to identify the attributes associated with each node. The demographic categories included were age, sex, gender, employment, ethnicity, and type of treatment. The output of a matrix coding query is a chart that displays the number of references coded at each node and the corresponding demographic attributes for each participant.

\section{Quantitative data analyses methods}

Univariate exploratory analyses were conducted to identify statistical differences among the groups in their desired treatment outcomes. The themes used in these analyses were derived from the completed Nvivo analysis of the free text goals. A chi-square analysis was completed for each Nvivo identified treatment outcome (stop MAT, avoid illicit drugs, live a "normal" life, manage pain, prevent OUD symptoms, taper off MAT, no changes in treatment) with age, sex, gender, ethnicity, employment, type of treatment, and source of first exposure to opioids (licit vs. illicit). An alpha of 0.05 was used to establish significance. All analyses had a degree of freedom (df) of 1 and created a $2 \times 2$ output. The associated phi value $(\varphi)$ was reported for these analyses. Age had a df of 2. For these analyses, Cramer's V value was reported.

\section{Ethics statement}

The study was approved by the Hamilton integrative Research Ethics Board (HiREB \#4556). All patients provided written informed consent.

\section{Results}

\section{Study participants' characteristics}

A total of 2,032 participants were recruited for this study. One participant had treatment goal data missing, which 
resulted in a sample of 2,031 participants $(1,135$ males, 896 females, and one intersex) whose treatment goals were analyzed qualitatively. The mean age was 39.1 years, $71.3 \%$ were of European ethnicity, and $66.2 \%$ were not currently working. Demographic details are presented in Table 1. Most participants had at least one positive urine drug screen for illicit opioids while on MAT (68.2\%), and $44.1 \%$ were first exposed to opioids through licit means (i.e., they were prescribed opioids for medical reasons).

\section{Objective 1: Qualitative patient important outcome data results}

Seven major themes were identified using Nvivo analysis, in order of frequency:

1. Stop MAT (includes stop methadone or buprenorphine/ naloxone treatment completely or to not be dependent on MAT);

2. Avoid illicit drugs (includes wanting to get clean, stay clean, abstinence, or sobriety from a variety of drugs, not just opioids);

3. Live a "normal" life (includes wanting a stable life, normal life, education, job or work, good mental health, or wanting to support their family or stay alive);

4. Manage pain (includes chronic pain or pain management);

5. Prevent OUD symptoms (includes withdrawal and craving);

6. Taper off MAT (includes wanting to taper off, wean off, or reduce dose);

7. No changes in treatment (includes keep everything as is, stabilize the dose, or nothing to add).

Table 1 Demographic characteristics $(n=2,031)$

\begin{tabular}{lc}
\hline Characteristic & $39.1(10.7)$ \\
Age (years), mean (SD) & 44.0 \\
Sex (female) & 71.3 \\
Ethnicity (European) & 33.8 \\
Currently employed & \\
& \\
Marital status & 50.4 \\
$\quad$ Never married & 28.9 \\
Currently married/common-law & 20.7 \\
Separated/divorced/widowed & \\
& \\
Level of education & 28.3 \\
$\quad$ None/elementary school & 43.1 \\
High school & 2.5 \\
Trade school & 25.7 \\
College/university & 0.4 \\
Postgraduate & \\
$\quad$ & \\
Details of opioid use & $24.8(9.25)$ \\
Age onset (years), mean (SD) & $54.5(63.1)$ \\
$\quad$ Treatment duration (months) (SD) & $70.4(41.3)$ \\
$\quad$ Methadone dose (mg/day), mean (SD) \\
Buprenorphine/naloxone dose (mg/day), \\
$\quad$ mean (SD) & $12.0(6.73)$ \\
Participants with at least one positive opioid urine & \\
$\quad$ screen in past 3 months & 68.2 \\
\hline Data presented as percentage, unless otherwise specified. \\
SD = standard deviation.
\end{tabular}

Participants were free to provide multiple desired treatment outcomes; therefore, the total number of responses exceeds the number of participants. Participants who had goals corresponding to both the stop MAT treatment and the taper off MAT treatment themes were grouped under the stop MAT treatment theme and removed from the taper off MAT treatment theme. These themes were separated as one implies getting off the program entirely (stop MAT), while the other theme implies, they may stay on the program, but at a lower dose. This resulted in the total number of responses decreasing from 3,310 to 3,020 .

Figure 1 shows the distribution of the seven different outcomes. The most desired goal was to stop MAT $(39 \%$ of responses), followed by avoiding illicit drugs (25\%), whereas the lowest percentage was for the goal to have no changes in treatment ( $4 \%$ of responses).

\section{Objective 2: Distribution of patient-important outcomes by predefined groups}

Patient responses were analyzed in comparison with age, sex, gender, ethnicity, employment and treatment duration, and type. Results are shown below.

\section{Age}

There were 203 responses from youths, 2,780 from adult, and 37 from seniors (Figure 2). The most common goal for all three age groups was to stop treatment (youth, 39.9\%; adults, $38.6 \%$; seniors, $32.4 \%$ ). The least common goal for the youth group was pain management $(1.5 \%)$.

\section{Sex}

The most common goal for both female and male participants was to stop treatment (females, 39.6\%; males, $37.8 \%$ ) (Figure 3). To live a normal life was the one response for intersex (100\%).

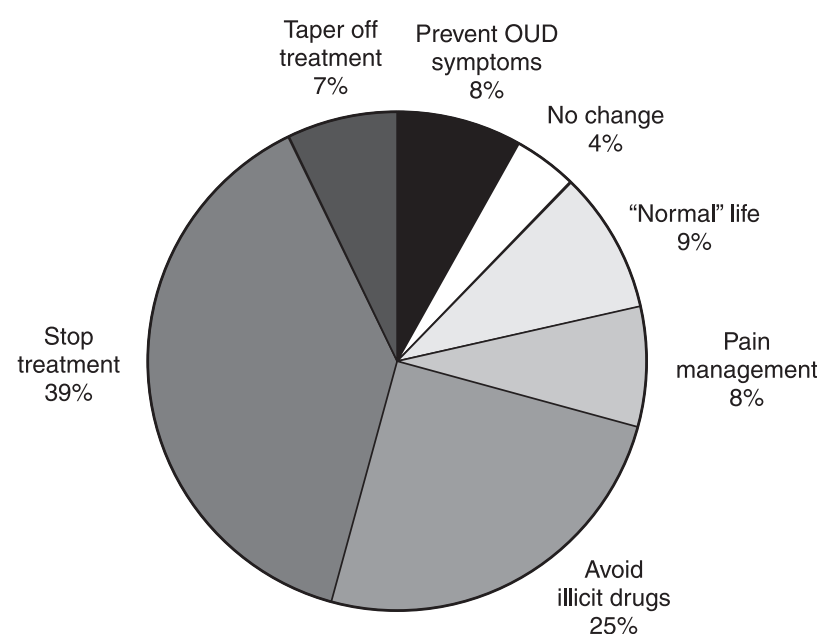

Figure 1 Percentage of responses per patient-important outcome group. OUD = opioid use disorder. 


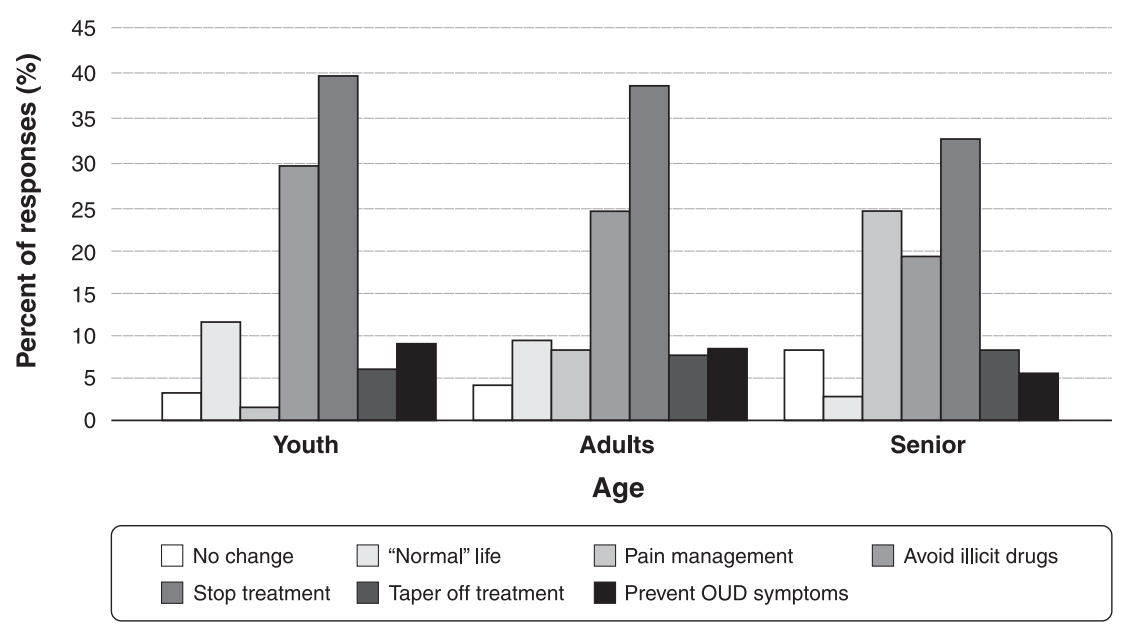

Figure 2 Desired treatment outcomes by age group. OUD = opioid use disorder.

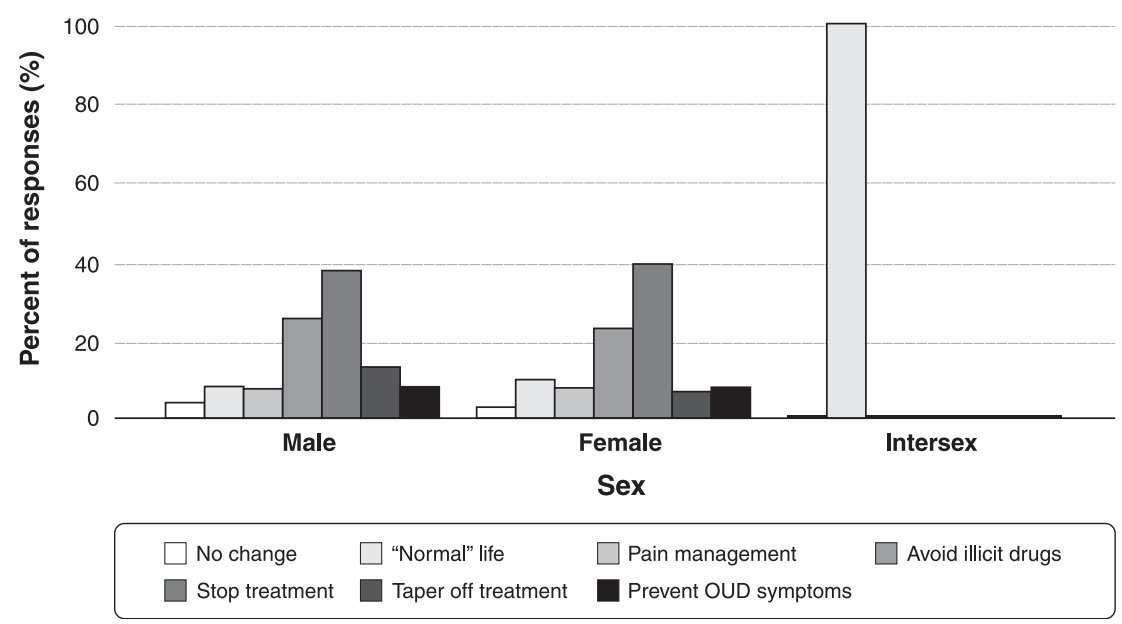

Figure 3 Sex differences in patient-important outcomes. OUD = opioid use disorder.

\section{Gender}

There were a total of 1,351 cisgender female responses, 1,646 cisgender male responses, and 23 other responses. The most common goal for both cisgender female and cisgender male participants was to stop treatment (cisgender females, 39.7\%; cisgender males, $37.7 \%$ ). The most frequent goal identified by participants grouped under the "other" category was to stop treatment (39.1\%).

\section{Ethnicity}

The majority of participants were European $(n=2,154)$ followed by "other" $(n=437)$ and Indigenous $(n=367)$ (Figure S1, available as online-only supplementary material). The most common goal for all ethnicities was to stop treatment.

\section{Employment}

The highest reported outcome by both unemployed and employed participants was to stop treatment (unemployed, 36.6\%; employed, 42.7\%). (Figure 4). The greatest difference in response by employment was seen in the pain management theme (unemployed, 9.47\%; employed, $4.78 \%$ ).

\section{Type of treatment}

There were 2,399 responses corresponding to methadone treatment, 616 responses relating to buprenorphine/ naloxone treatment, and four responses for other forms of treatment (Figure 5). The most common goal for both methadone and buprenorphine/naloxone treatment was to stop treatment (methadone, 38.2\%; buprenorphine/ naloxone, $40.1 \%$ ).

\section{Length of treatment}

The most common goal at all lengths of treatment was to stop treatment (1 year or less, 34.2\%; $1-5$ years, $40.5 \%$; 5 -10 years, $42.6 \%$; $10-15$ years, 36\%; $15+$ years, $41.4 \%$ ) (Figure S2, available as online-only supplementary material). 


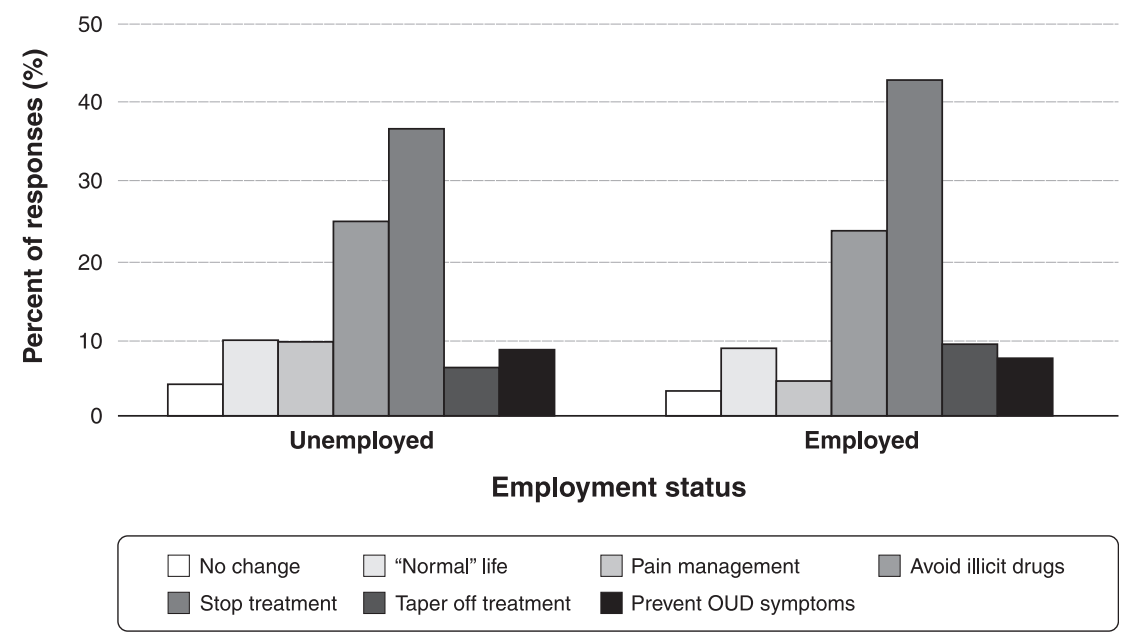

Figure 4 Patient-important outcomes by employment status. OUD = opioid use disorder.

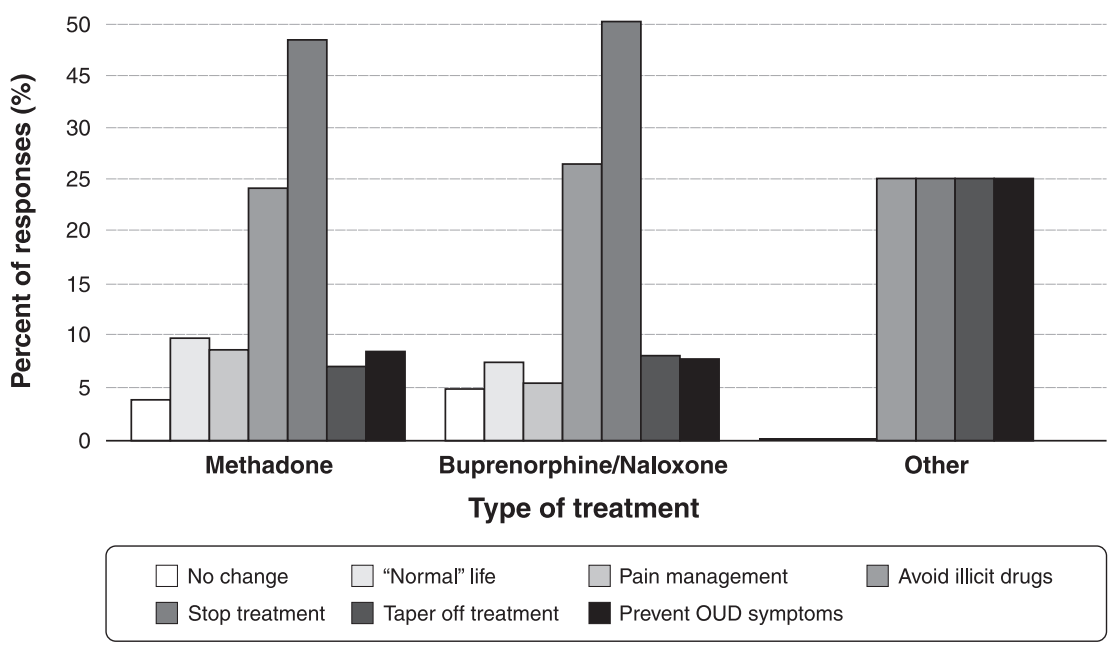

Figure 5 Differences in type of treatment seen in patient-important outcomes. OUD = opioid use disorder.

First exposure to opioids: legitimately prescribed (licit) vs. recreational exposure (illicit)

The most common goal, regardless of the source of first exposure to opioids, was to stop treatment (licit, 37.9\%; illicit, $39.1 \%$ ). Participants who were first exposed to opioids through licit means had more responses listing pain management as their goal compared to those who were first exposed to opioids through illicit means (licit, $12.4 \%$; illicit, $4.3 \%$ ) (Figure S3, available as online-only supplementary material).

\section{Correlation analyses}

Univariate exploratory analyses to identify statistical differences among the groups in the outcomes they identified as important showed that all groups had stop MAT and avoid illicit drugs as the leading treatment goals. However, some differences among groups were also observed. Specifically, the following associations were found to be significant: pain management and age $(p \leqslant 0.001)$, stop MAT and sex $(p=0.047)$, stop MAT and ethnicity $(p=0.001)$, taper off MAT and ethnicity $(p=0.007)$, pain management and employment $(p \leqslant 0.001)$, stop MAT and employment $(p=0.013)$, taper off MAT and employment $(p=0.008)$, live a "normal" life and type of treatment $(p=0.030)$, pain management and type of treatment $(p=0.005)$, pain management and source of first exposure to opioids ( $p \leqslant 0.001)$, and live a "normal" life and source of first exposure to opioids $(p=0.021)$ (for additional details, see Table S1, available as online-only supplementary material).

\section{Discussion}

In this large study of 2,031 patients with OUD, we identified that $39 \%$ of patients wanted to stop MAT and $25 \%$ wanted to stop all drugs, not just opioids. Yet, current MAT programs are focused on treatment retention and stopping or reducing illicit opioid use. This suggests that $64 \%$ of patients in this cohort are not meeting treatment goals for traditional MAT programs. This may be an important consideration when assessing MAT effectiveness measures, as well as considering individual patient 
preferences based on sociodemographic factors and personalized medicine.

Patients of all ages wanted to stop MAT and avoid illicit drugs. While older adults had pain management as their second most frequent goal, the majority of patients regardless of their sociodemographic variables - wanted to stop or taper off MAT.

Current OUD management guidelines recommend the use of MAT to manage OUD; however, these guidelines do not include patient-related goals and do not specify the length of time for which MAT should be considered. ${ }^{19}$ In this study, patients' most frequently reported goal of OUD treatment was to stop MAT (39\%). However, in the absence of recommendations based on evidence from clinical trials on the duration of MAT and the desire of patients to stop MAT, treatment adherence and the prognosis of OUD are unlikely to be favorable.

Guidelines also strongly recommended that withdrawal management only without transition to a MAT should not be used in managing OUD, ${ }^{19}$ as this is suggested to be associated with relapse, overdose, and risk of unsafe substance use compared to no treatment at all, while patient-important goals identified in our study stated that only $8 \%$ of responses were related to OUD symptoms management. Most participants in this study had at least one positive urine drug screen for opioid while on MAT $(68.2 \%)$ during the preceding 3 months, despite being on MAT for an average of 4.5 years. The risk of relapse and overdose are real challenges in OUD, but many trials use short-term, narrowly focused outcome measures, such as urine drug screens, to determine treatment effectiveness. If efficacy of MAT is based on opioid-negative urine drug screens, then MAT is ineffective in $68 \%$ of patients in this study. The use of urine drug screens to measure the effectiveness of MAT in clinical trials fails to capture important outcomes associated with the chronicity of OUD, which limits the scope of treatment.

A frequently mentioned treatment goal $(25 \%)$ was to avoid all illicit drugs, not just opioids. We previously reported that comorbid substance use in this population is common, with $42 \%$ having a comorbid substance use disorder. ${ }^{20}$ Despite this, clinical trials of MAT for OUD exclude patients with co-substance use. ${ }^{14}$ This exclusion is leaving a significant proportion of patients with OUD with unmet needs and unmeasured treatment outcomes.

Another factor we explored that may influence patients' treatment goals is the type of MAT prescribed. In this study, we reported patient-important goals by the type of MAT they are receiving. Patients' desire to be off treatment may be explained by the stigma attached to methadone. ${ }^{21}$ However, the results of this study showed that patients on other MAT also wanted to be off treatment. Therefore, stigma alone may not explain why the most frequent patient-important outcome is to stop treatment.

Our findings also suggest that patients who were first exposed to opioids through licit vs. illicit means may have different goals to achieve out of MAT. We found that those who were exposed to opioids through licit means were significantly more likely to have pain management as a goal, perhaps because their first exposure to opioids was probably for pain management. In addition, MAT, including methadone, is used for pain management; therefore, it is expected that patients with chronic pain conditions may wish to continue using MAT to relieve pain. Additionally, those that were introduced to opioids through illicit means were likely to list "live a normal life" as a goal. Previous research that has looked into the sources of introduction to opioids has found differences in substance use and demographic characteristics in those introduced to opioids by prescription vs. other means. ${ }^{22,23}$ This suggests that participants who were introduced to opioids through illicit means may have vulnerability factors for substance use disorder, such as novelty-seeking and risk-taking behavior, compared to people with pain who were prescribed opioids to manage it and would be more likely to have treatment goals pertaining to stability/living a normal life. ${ }^{23}$

Although the reasons why patients wanted to be off MAT cannot be explained in this study, a treatment plan that includes patient-important goals and evidencebased, informed precision medicine is needed to improve treatment outcomes in OUD. While it may seem challenging to achieve a consensus between patients and treatment programs on what constitutes a good treatment outcome, previous studies showed that it is possible to obtain such agreement. ${ }^{24}$ Nevertheless, there is a lack of patient-important and patient-identified outcome sets in clinical research and practice. ${ }^{25}$ No previous work on patient-important outcomes in OUD to inform clinical trials has been completed, despite the ongoing opioid crisis.

Comparisons of treatment plans and goals vary greatly across clinical care settings, patients' expectations, and services delivered. ${ }^{26}$ For example, the duration of treatment may have an impact on patient engagement in services whose patients perceive these services as more helpful than short-term treatment. ${ }^{27}$ Furthermore, patients' suggestions on their treatment goals often differ from their clinicians' opinions. One study found that patients with addiction saw physical health as a goal more often than their clinicians did. ${ }^{28}$ Thus, patient and clinician communication about the goals and expectations of treatment may be beneficial to translate patients' opinions and choices of what constitutes a relevant outcome for them into the course of treatment. Communication may also help patients' positive opinions on long-term goals become a part of their service plan, potentially leading to achievable goals. This concept was summarized by stating that limiting discrepancies between patients' and clinicians' goals of addiction service might lead to convergence, which is likely necessary for positive treatment goals and better care of patients with addiction. ${ }^{28}$

Discrepancies are often related to the concept that existing treatments and clinical trials in OUD have used convenience outcomes that are objectively measurable (such as urine drug screens) without consideration for patient-important outcomes, sociodemographic differences, and patients' goals or group differences. Additionally, guidelines also indicate that there is little consistent evidence to evaluate the effectiveness of OUD treatment. ${ }^{29}$ Reviews evaluating OUD treatment effectiveness have found great variability in the selected goals between studies, ${ }^{30-32}$ leading to difficulty in establishing a 
real treatment effect. Each study measures a different set of goals that define success in arbitrary or accessible terms, limiting cross-study comparisons. This is an important limitation in addiction research that must be overcome if a consensus on what works for OUD management and how to assign a treatment goal is to be achieved.

Despite being the largest study to date and including unrestricted responses from patients receiving active treatment, some limitations of our work should be considered. The study cohort may not be representative of all patients with OUD, as there is an expected self-selection bias in voluntary participation in research compared to those who do not participate. The study findings may not be generalizable to the entire Canadian population, as our study sample was recruited from community clinics in the province of Ontario. It is important to note that our mean age and sex distribution resembles data collected by Public Health Ontario in 2018, where age groups and sex distribution were similar to those of the study participants. $^{33}$

Other limitations to consider are other variables that may play a role in determining patient-oriented goals and which are not measured in this study, such as personality type. Previous research suggests that there may be a relationship between specific traits and chronic substance use. ${ }^{34}$ There is also the possibility that patients who no longer attend treatment programs and achieved sustainable recovery may have a different outlook on treatment goals compared to patients in the active phase of substance use disorder. Despite these limitations, the responses provided by 2,031 patients in active treatment are important findings that at least will apply to a similar population in the active phase of the disorder.

In conclusion, in this mixed-methods study, we analyzed answers to an open-ended question - letting participants express their opinions without any constraints on the type, length, or direction of the answer - on what participants wanted out of treatment for OUD. We identified patient-important outcomes for OUD that may inform future trials of MAT for OUD. Despite implementation of many different measures, opioid use has not seen adequate control. Therefore, identifying effective ways to manage OUD remains both urgent and timely. Treatment guidelines and programs rely on well-conducted clinical trials; when these begin to include patientimportant outcomes, their results may lead to a paradigm shift in what treatments outcomes should be considered, what medications are truly effective, for what goal these results apply and to what patients, and how treatment programs should be evaluated when it comes to resource allocations and policy making. We need a shift in how these treatments are tested for effectiveness to incorporate patient-important outcomes and provide a precision medicine approach to managing the OUD epidemic.

\section{Acknowledgements}

This work was supported by the Canadian Institute for Health Research (CIHR; award 156306).

\section{Disclosure}

The authors report no conflicts of interest.

\section{References}

1 Rudd RA, Aleshire N, Zibbell JE, Gladden RM. Increases in drug and opioid overdose deaths -- United States, 2000-2014. MMWR Morb Mortal Wkly Rep. 2016;64:1378-82.

2 Zhong W, Maradit-Kremers H, Sauver JL, Yawn BP, Ebbert JO, Roger VL, et al. Age and sex patterns of drug prescribing in a defined American population. Mayo Clin Proc. 2013;88:697-707.

3 Simoni-Wastila L, Ritter G, Strickler G. Gender and other factors associated with the nonmedical use of abusable prescription drugs. Subst Use Misuse. 2004;39:1-23.

4 Porter J, Jick $\mathrm{H}$. Addiction rare in patients treated with narcotics. N Engl J Med. 1980;302:123.

5 DeWeerdt S. Tracing the US opioid crisis to its roots. Nature. 2019; 573:S10-12.

6 EF McCance-Katz; Substance Abuse and Mental Health Services Administration (SAMHSA). The national survey on drug use and health: 2017. [cited 2020 May 4]. https://www.google.com/url?sa=t\&rct $=j \& q=\&$ esrc $=$ s $\&$ source $=$ web $\&$ cd $=\& v e d=2$ ahUKEwiKmMWdr9TpAhWoP OwKHb8tAEkQFjABegQIAhAC\&url=https\%3A\%2F\%2Fwww.samhsa. gov\%2Fdata\%2Fsites\%2Fdefault\%2Ffiles\%2Fnsduh-ppt-09-2018.pdf \&usg=AOvVaw3W0-cJVaTnczJNmZc-WUTN

7 American Psychiatric Association. Diagnostic and Statistical Manual of Mental Disorders, Fifth Edition (DSM-5).Arlington: American Psychiatric Publishing; 2013.

8 Cicero TJ, Ellis MS. The prescription opioid epidemic: a review of qualitative studies on the progression from initial use to abuse. Dialogues Clin Neurosci. 2017;19:259-69.

9 Schuckit MA. Treatment of opioid-use disorders. N Engl J Med. 2016;375:357-68.

10 Wright NM, Sheard L, Adams CE, Rushforth BJ, Harrison W, Bound $\mathrm{N}$, et al. Comparison of methadone and buprenorphine for opiate detoxification (LEEDS trial): a randomised controlled trial. $\mathrm{Br} \mathrm{J}$ Gen Pract. 2011;61:e772-80.

11 Bertschy G. Methadone maintenance treatment: an update. Eur Arch Psychiatry Clin Neurosci. 1995;245:114-24.

12 Lions C, Carrieri MP, Michel L, Mora M, Marcellin F, Morel A, et al. Predictors of non-prescribed opioid use after one year of methadone treatment: an attributable-risk approach (ANRS-Methaville trial). Drug Alcohol Depend. 2014;135:1-8.

13 Dennis BB, Sanger N, Bawor M, Naji L, Plater C, Worster A, et al. A call for consensus in defining efficacy in clinical trials for opioid addiction: combined results from a systematic review and qualitative study in patients receiving pharmacological assisted therapy for opioid use disorder. Trials. 2020;21:30.

14 Dennis BB, Roshanov PS, Naji L, Bawor M, Paul J, Plater C, et al. Opioid substitution and antagonist therapy trials exclude the common addiction patient: a systematic review and analysis of eligibility criteria. Trials. 2015;16:475.

15 statistics Canada. StatCan Blog [Internet]. Statistics Canada; 2013. https://www150.statcan.gc.ca/n1/pub/12-581-x/12-581-x2013000-eng. htm

16 Castleberry A. NVivo 10 [software program]. Version 10. QSR International; 2012. Am J Pharm Educ. 2014;78:25.

17 Zamawe FC. The implication of using NVivo software in qualitative data analysis: Evidence-based reflections. Malawi Med J. 2015;27:13-5.

18 Feng $\mathrm{X}$, Behar-Horenstein L. Maximizing NVivo utilities to analyze open-ended responses. Qual Rep. 2019;24:563-71.

19 Bruneau J, Ahamad K, Goyer MÈ, Poulin G, Selby P, Fischer B, et al. Management of opioid use disorders: a national clinical practice guideline. CMAJ. 2018;190:E247-57.

20 Rosic T, Naji L, Bawor M, Dennis BB, Plater C, Marsh DC, et al. The impact of comorbid psychiatric disorders on methadone maintenance treatment in opioid use disorder: a prospective cohort study. Neuropsychiatr Dis Treat. 2017;13:1399-1408.

21 Woo J, Bhalerao A, Bawor M, Bhatt M, Dennis B, Mouravska N, et al. "Don't judge a book by its cover": a qualitative study of methadone patients' experiences of stigma. Subst Abuse. 2017;11:11782218 16685087. 
22 Tsui Jl, Herman DS, Kettavong M, Alford D, Anderson BJ, Stein MD. Physician introduction to opioids for pain among patients with opioid dependence and depressive symptoms. J Subst Abuse Treat. 2010; 39:378-83.

23 Madruga CS, Laranjeira R, Caetano R, Pinsky I, Zaleski M, Ferri CP. Use of licit and illicit substances among adolescents in Brazil-a national survey. Addict Behav. 2012;37:1171-5.

24 Noble AJ, Marson AG. Which outcomes should we measure in adult epilepsy trials? The views of people with epilepsy and informal carers. Epilepsy Behav. 2016;59:105-10.

25 Turk DC, Dworkin RH, Revicki D, Harding G, Burke LB, Cella D, et al. Identifying important outcome domains for chronic pain clinical trials: an IMMPACT survey of people with pain. Pain. 2008;137: 276-85.

26 McLellan AT, Lewis DC, O'Brien CP, Kleber HD. Drug dependence, a chronic medical illness: implications for treatment, insurance, and outcomes evaluation. JAMA. 2000;284:1689-95.

27 Nordfjaern T, Rundmo T, Hole R. Treatment and recovery as perceived by patients with substance addiction. J Psychiatr Ment Health Nurs. 2010;17:46-64.

28 Joosten EAG, De Weert-Van Oene GH, Sensky T, Van Der Staak CP, De Jong CA. Treatment goals in addiction healthcare: the perspectives of patients and clinicians. Int J Soc Psychiatry. 2011;57:263-76.
29 Smith CBR, Brands B, Lacroix S, Weisdorf T, Novotná G, Kushnir V. Methadone maintenance treatment program standards and clinical guidelines (College of Physicians and Surgeons of Ontario) [Internet] 2011 Feb [cited 2020 May 4]. https://www.researchgate.net/publication/ 271076438_Methadone_Maintenance_Treatment_Program_Standards_ and_Clinical_Guidelines_College_of_Physicians_and_Surgeons_of_ Ontario

$30 \mathrm{Li}$ Y, Kantelip JP, Gerritsen-van Schieveen P, Davani S. Interindividual variability of methadone response: impact of genetic polymorphism. Mol Diagn Ther. 2008;12:109-24.

31 Mattick RP, Breen C, Kimber J, Davoli M. Methadone maintenance therapy versus no opioid replacement therapy for opioid dependence. Cochrane Database Syst Rev. 2009;(3):CD002209.

32 Hedrich D, Alves P, Farrell M, Stöver H, Møller L, Mayet S. The effectiveness of opioid maintenance treatment in prison settings: a systematic review. Addiction. 2012;107:501-17.

33 Public Health Ontario. Interactive opioid tool: opioid-related morbidity and mortality in Ontario [Internet]. 2019 [cited 2020 May 4]. https:// www.publichealthontario.ca/en/data-and-analysis/substance-use/inter active-opioid-tool\#/ageSex.

34 Ersche KD, Turton AJ, Pradhan S, Bullmore ET, Robbins TW. Drug addiction endophenotypes: impulsive versus sensation-seeking personality traits. Biol Psychiatry. 2010;68:770-3. 